\title{
"That was then, this is now" improving public health syndromic surveillance baselines
}

\author{
Roger Morbey*, Alex J. Elliot, Paul Loveridge, Helen Hughes, Sally Harcourt and \\ Sue Smith
}

Public Health England, Birmingham, United Kingdom

\section{Objective}

To improve the ability of syndromic surveillance systems to detect unusual events.

\section{Introduction}

Syndromic surveillance systems are used by Public Health England (PHE) to detect changes in health care activity that are indicative of potential threats to public health. By providing early warning and situational awareness, these systems play a key role in supporting infectious disease surveillance programmes, decision making and supporting public health interventions.

In order to improve the identification of unusual activity, we created new baselines to model seasonally expected activity in the absence of outbreaks or other incidents. Although historical data could be used to model seasonality, changes due to public health interventions or working practices affected comparability. Specific examples of these changes included a major change in the way telehealth services were provided in England and the rotavirus vaccination programme introduced in July 2013 that changed the seasonality of gastrointestinal consultations. Therefore, we needed to incorporate these temporal changes in our baselines.

\section{Methods}

We used negative binominal regression to model daily syndromic surveillance, allowing for day of week and public holiday effects. To account for step changes in data caused by changes in healthcare system working practices or public health interventions we introduced specific independent variables into the models. Finally, we smoothed the regression models to provide short term forecasts of expected trends.

The new baselines were applied to PHE's four syndromic surveillance systems for daily surveillance and public-facing weekly bulletins.

\section{Results}

We replaced traditional surveillance baselines (based on simple averages of historical data) with the regression models for daily surveillance of 53 syndromes across four syndromic surveillance systems. The improved models captured current seasonal trends and more closely reflected actual data outside of outbreaks.

\section{Conclusions}

Syndromic surveillance baselines provide context for epidemiologists to make decisions about seasonal disease activity and emerging public health threats. The improved baselines developed here showed whether current activity was consistent with expected activity, given all available information, and improved interpretation when trends diverged from expectations.

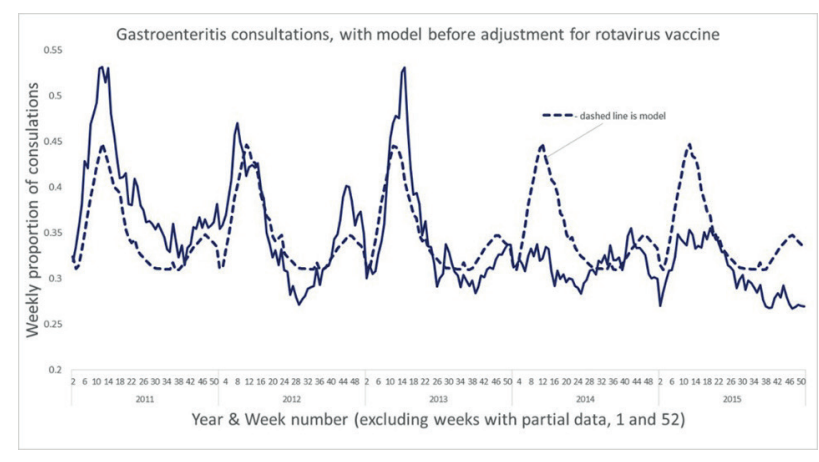

Keywords

syndromic; surveillance; Public Health

\section{Acknowledgments}

RAM, AJE \& GES are partly funded by the National Institute for Health Research (NIHR) Health Protection Research Unit in Emergency Preparedness and Response. The views expressed are those of the authors and not necessarily those of the National Health Service, the NIHR, the Department of Health or Public Health England.

\section{${ }^{*}$ Roger Morbey}

E-mail: roger.morbey@phe.gov.uk 